\title{
Multiscale analysis of morphology and mechanics in tail tendon from the ZDSD rat model of type 2 diabetes
}

\author{
Armando Diaz Gonzalez ${ }^{a}$, Maxime A. Gallant ${ }^{b}$, David B. Burra,b, and Joseph M. Wallace ${ }^{a, c,{ }^{*}}$ \\ aDepartment of Biomedical Engineering, Indiana University-Purdue University at Indianapolis, \\ Indianapolis, IN, USA \\ bDepartment of Anatomy and Cell Biology, Indiana University School of Medicine, Indianapolis, \\ IN, USA \\ 'Department of Orthopaedic Surgery, Indiana University School of Medicine, Indianapolis, IN, \\ USA
}

\begin{abstract}
Type 2 diabetes (T2D) impacts multiple organ systems including the circulatory, renal, nervous and musculoskeletal systems. In collagen-based tissues, one mechanism that may be responsible for detrimental mechanical impacts of T2D is the formation of advanced glycation end products (AGEs) leading to increased collagen stiffness and decreased toughness, resulting in brittle tissue behavior. The purpose of this study was to investigate tendon mechanical properties from normal and diabetic rats at two distinct length scales, testing the hypothesis that increased stiffness and strength and decreased toughness at the fiber level would be associated with alterations in nanoscale morphology and mechanics. Individual fascicles from female Zucker diabetic SpragueDawley (ZDSD) rats had no differences in fascicle-level mechanical properties but had increased material-level strength and stiffness versus control rats (CD). At the nanoscale, collagen fibril Dspacing was shifted towards higher spacing values in diabetic ZDSD fibrils. The distribution of nanoscale modulus values was also shifted to higher values. Material-level strength and stiffness from whole fiber tests were increased in ZDSD tails. Correlations between nanoscale and microscale properties indicate a direct positive relationship between the two length scales, most notably in the relationship between nanoscale and microscale modulus. These findings indicate that diabetes-induced changes in material strength and modulus were driven by alterations at the nanoscale.
\end{abstract}

\section{Keywords}

AFM; Tension; Nanoscale; Ultrastructure; Modulus

\footnotetext{
(C) 2013 Elsevier Ltd. All rights reserved

*Corresponding Author: Dr. Joseph M. Wallace Indiana University-Purdue University at Indianapolis Department of Biomedical Engineering 723 W Michigan St. SL220D Indianapolis, IN 46202 Phone: (317) 274-2448 Fax: (317) 278-2455 jmwalla@iupui.edu.

Publisher's Disclaimer: This is a PDF file of an unedited manuscript that has been accepted for publication. As a service to our customers we are providing this early version of the manuscript. The manuscript will undergo copyediting, typesetting, and review of the resulting proof before it is published in its final citable form. Please note that during the production process errors may be discovered which could affect the content, and all legal disclaimers that apply to the journal pertain.

CONFLICT OF INTEREST:

We wish to confirm that there are no known conflicts of interest associated with this publication and there has been no significant financial support for this work that could have influenced its outcome.
} 


\section{INTRODUCTION}

The effects of diabetes are felt worldwide across all age groups, ethnicities and genders. In 2011, an estimated 25.8 million Americans suffered with effects of diabetes resulting in direct care expenditures of $\$ 120$ billion (Centers 2011). In adults, $90-95 \%$ of cases are Type 2 diabetes (T2D, previously non-insulin-dependent diabetes mellitus). T2D usually begins as insulin resistance, and has detrimental impacts on multiple organ systems including the circulatory, renal and nervous systems. Destructive effects are also present in the musculoskeletal system, although studies are limited by comparison.

Several rodent models of T2D exist including the Zucker Diabetic Fatty (ZDF) rat and the Zucker diabetic Sprague-Dawley (ZDSD) rat (Reinwald et al. 2009). The primary advantage of ZDSD over the ZDF model is that the diabetes induced is not leptin-dependent. ZDSD is more translatable to the human condition, which is also not leptin dependent. Moreover, ZDSD rats gradually develop a T2D-like condition as adults in response to chronic dietary manipulation, whereas onset in ZDF is rapid and begins at an early age. Consequently, skeletal fragility in the ZDF rats is compounded by structural deficits that occur during growth, a situation not found in humans with adult onset T2D. The onset of diabetes in ZDSD rats occurs after 6 months of age in females, when the animals are considered to be fully-adult. Thus, the key factors responsible for the onset and progression of T2D in ZDSD are more similar to human T2D.

Tendon has a hierarchical structure based on Type I collagen (hereafter, referred to as collagen (Kastelic et al. 1978)). Collagen is the principal source of tensile strength in many tissues (Canty and Kadler 2005) and may provide some degree of toughness (Launey et al. 2010). Procollagen is a heterotrimeric molecule composed of three helical a chains wound into a right handed triple helix. Once procollagen molecules are secreted, non-helical propeptide ends are cleaved releasing a mature tropocollagen molecule. A three dimensional collagen fibril forms as tropocollagen self-assembles into a staggered, parallel twist (Hodge and Petruska 1963; Orgel et al. 2006).

T2D affects the quality of collagen-based tissues through multiple mechanisms. One such mechanism is the formation of advanced glycation end products (AGEs) (Singh et al. 2001). Enzymatic crosslinking is tightly regulated process (Knott and Bailey 1998) and is positively correlated with mechanical integrity (Fratzl 2008). The reduction in tissue quality with T2D is likely the result of chronic hyperglycemia increasing the presence of AGEs which form when reducing sugars react with free amino groups in proteins. These reactions occur randomly with lysine or arginine residues anywhere in collagen molecules, forming permanent non-enzymatic crosslinks (Singh et al. 2001; Merlotti et al. 2010). AGEs can accumulate with time and because of the slow turnover in collagen-based tissues, lead to significant functional impairment including increased stiffness and decreased toughness, resulting in brittle behavior (Vashishth et al. 2001; Reddy 2004; Tang et al. 2007). However, the association between diabetes, AGEs and mechanical properties in collagen based-tissues is an unresolved and highly contentious debate, as many studies contradict these differences (Duquette et al. 1996; Couppe et al. 2009; Fox et al. 2011).

Collagen fibrils possess a characteristic axial repeat pattern called the D-periodicity. Although postulated to have a singular value of $67 \mathrm{~nm}$ (Hodge and Petruska 1963), recent work has shown that $D$-spacing exists with a distribution of values (Wallace et al. 2010a). This distribution changes with several disease states in bone (Wallace et al. 2010a; Wallace et al. 2011; Warden et al. 2013), skin (Fang et al. 2012) and tendon (Kemp et al. 2012), suggesting a metric that may represent a diagnostic option for diseases in collagen-based tissues. To put functional meaning behind this measure of nanoscale morphology, recent 
work focused on mechanically probing collagen fibrils (Kemp et al. 2012; Wallace et al. 2012). These studies showed nanoscale effects of disease in individual fibrils. However, the studies were limited in scope and failed to connect nanoscale changes to higher length scales.

Few studies have investigated the collagen ultrastructure in diabetic tissues (Odetti et al. 2000; Svensson et al. 2013) and how nanoscale features relate to more clinically-relevant length scales (Rigozzi et al. 2012; Li et al. 2013). The purpose of this study was to investigate mechanical properties of tail tendon fascicles from normal and diabetic rats at two distinct length scales, testing the hypothesis that increased stiffness and strength and decreased toughness at the fiber level are associated with alterations in nanoscale morphology and mechanics.

\section{MATERIALS AND METHODS}

\section{Animals and tissue collection}

Female Zucker diabetic Sprague-Dawley (ZDSD, PreClinOmics, Indianapolis) and control rats (CD: Sprague-Dawley-derived, Charles River Laboratory) were used with prior approval (IACUC \#3146). At 20 weeks of age, animals were switched from regular chow (Purina 5008) to a high fat test diet (Test diet 5SCA), a change which induces, synchronizes and maintains T2D in ZDSD rats. Rats remained on the high fat diet until sacrifice by $\mathrm{CO}_{2}$ inhalation at 32 weeks of age. Blood glucose levels were measured to verify a diabetic state in the ZDSD rats (greater than $250 \mathrm{mg} / \mathrm{dl}$ ). Of the five ZDSD rats used, only four became diabetic and were included. The proximal end of the tail of each rat ( $25 \%$ of the total length) was removed, wrapped in phosphate buffered saline (PBS)-soaked gauze and stored at $-20^{\circ} \mathrm{C}$ for nanoscale testing. The remaining $75 \%$ of the tail was similarly stored for microscale mechanical testing.

\section{Atomic Force Microscopy (AFM) Imaging}

Fascicles were removed and processed for AFM imaging and indentation as previously described (Kemp et al. 2012). Briefly, each fascicle was rinsed in water, placed on a glass slide and flattened with forceps. The sample was allowed to dry just enough to adhere to the glass, then was submerged in water and imaged in peak force tapping mode (Bruker Catalyst AFM, ScanAsyst Fluid+ probes). Prior to imaging, each probe was calibrated for indentation. Deflection sensitivity $(\mathrm{nm} / \mathrm{V})$ was measured by pushing the probe into a glass surface, and the spring constant $(\mathrm{N} / \mathrm{m})$ was determined using the thermal tuning method.

To measure morphology, $5 \mu \mathrm{m}^{2}$ images were acquired from 2-3 locations in each of 2-3 fascicles per animal. D-periodic spacing was measured from individual fibrils using a two dimensional Fast Fourier Transform (2D FFT), as previously described (Wallace et al. 2010b; Wallace et al. 2011; Kemp et al. 2012; Erickson et al. 2013). Analysis was performed on 10-15 individual fibrils per location, an average of 110 fibrils per animal.

\section{AFM-Based Indentation}

After image acquisition, four to five locations along the length of individual fibrils were indented to $20 \mathrm{nN}$. Indents were at least $10 \mathrm{D}$-periodic units apart and targeted to the overlap region for consistency. On average, 70 individual fibrils were indented from each tail for $\sim 300$ total indentations per animal. Indentation depths are relatively deep despite the low applied force. Therefore, the Sneddon model of contact between a rigid cone and an elastic half space was used to calculate indentation modulus $\left(\mathrm{E}_{\mathrm{S}}\right)$ from the unloading curve of each indent. 


$$
F=\frac{2}{\pi} \mathrm{C} \frac{E_{s}}{1-v_{s}^{2}} \mathrm{Ctan} \alpha \mathrm{C} \delta^{2} \quad \text { equation } 1
$$

In equation $1, \mathrm{~F}$ is force and $\delta$ is deformation. $\mathrm{a}$ is the opening angle of the probe $\left(17.5^{\circ}\right)$. Poisson's ratio $\left(v_{\mathrm{s}}\right)$ is not fully understood for collagen, so a value of 0.35 was chosen for all samples (Kemp et al. 2012).

\section{Microscale testing of tendon fascicles}

From the distal portion of each tail, $90 \mathrm{~mm}$ lengths of tendon fascicles were removed and placed in PBS. At all times, the fascicles remained hydrated. The diameter of each fascicle was measured at five locations along its length at 100X using an inverted optical microscope, and the mean width from each fascicle was calculated as the average of these measurements. Assuming a circular cross section, cross sectional area was calculated using this mean width/diameter. Each end of the fascicle was sandwiched between two pieces of 180 grit sandpaper and placed within the grips of a testing system equipped with a $25 \mathrm{~N}$ load cell (Test Resources, Model 100R500-12, Shakopee, MN). Fascicles (10-12 per animal) were tested to failure in displacement control at $0.1 \mathrm{~mm} / \mathrm{sec}$. Force and displacement were recorded at $25 \mathrm{~Hz}$. Force was normalized by cross sectional area to obtain stress at each data point. Displacement was normalized by the original gauge length to calculate average strain across the gauge section. Yield was determined from the stress-strain curve using the $0.2 \%$ offset method. The slope of the linear region past the toe-in was calculated. A second line with this slope was offset from the first by $0.2 \%$ strain $(2000 \mu \varepsilon)$. Yield was defined as the point where this offset line crossed the data curve.

\section{Statistical Analysis}

All statistical analyses utilized SPSS (Version 19, IBM). A value of $\mathrm{p}<0.05$ was considered significant. Values measured from an individual tail were averaged, yielding a single value for each parameter for that sample. Because of the small sample sizes $(n=5$ for $C D, n=4$ for ZDSD), CD and ZDSD were compared using non-parametric Mann Whitney U Tests. To investigate differences in distributions of fibril morphology and indentation modulus, the Cumulative Distribution Function (CDF) of each group was computed and KolmogorovSmirnov (KS) tests were applied to the data. In an attempt to develop predictive relationships between microscale and nanoscale properties, Pearson product-moment correlations were calculated.

\section{RESULTS}

\section{General Animal Information}

At the beginning of the high fat diet, there were no differences in body weight, blood glucose or HbA1c between CD and ZDSD rats (Table 1). By the end of the 12 week high fat diet period, there was still no difference in body weight but ZDSD had significantly higher blood glucose $(\mathrm{p}=0.016)$ and Hb1 Ac $(\mathrm{p}=0.016)$ versus CD. At sacrifice, the lowest ZDSD glucose level was $420 \mathrm{mg} / \mathrm{dl}$ while the highest CD level was $129 \mathrm{mg} / \mathrm{dl}$.

\section{Morphological Changes in ZDSD Tail Tendon Fibrils}

The D-periodic spacing of individual fibrils was assessed as a function of the ZDSD disease state. All measurements from a single animal were averaged, and the mean values were $67.7 \pm 0.4 \mathrm{~nm}$ and $67.8 \pm 0.3 \mathrm{~nm}$ for $\mathrm{CD}(\mathrm{n}=5)$ and ZDSD $(\mathrm{n}=4)$, respectively. There was no effect of diabetes on mean fibril spacing $(\mathrm{p}=0.645)$. Figure 1 shows a boxplot representation 
of data from all fibrils within each group ( $\mathrm{n}=564$ for CD, $\mathrm{n}=440$ for ZDSD), although statistics were performed on the mean value from each tail.

When all measured fibrils in each group were analyzed, a distribution of D-period spacing values was present in both groups (Figure 2A). The ZDSD distribution was sharper and shifted to higher spacing values for the majority of its population versus $\mathrm{CD}$. Descriptive statistics echo these observations. ZDSD had a lower standard deviation (CD: $0.95 \mathrm{~nm}$, ZDSD: $0.80 \mathrm{~nm}$ ) and higher kurtosis (CD: 0.568, ZDSD: 2.289) indicating a sharper peak. Based on the histograms ( $1 \mathrm{~nm}$ bin sizes centered on whole integer values), curve fitting was performed using Gaussian distributions (data not shown). The full width at half max (a measure of the breadth of the distribution) was larger in CD $(2.28 \mathrm{~nm})$ versus ZDSD (1.51 $\mathrm{nm}$ ), further indicating that the ZDSD distribution was narrower. Finally, the skewness of the populations were negative (CD: -0.088, ZDSD: -0.717 ), indicating a shift towards higher values with a longer tail to the left. The skewness was larger in ZDSD, supporting the contention that the ZDSD population was shifted higher versus CD.

The Cumulative Distribution Function (CDF) was computed and shown to be significantly different between groups ( $\mathrm{p}<0.001$, Figure $2 \mathrm{~B}$ ). The CDFs further illustrate that the ZDSD population distribution was shifted to higher values versus $\mathrm{CD}$, driven by the slower rise in the ZDSD population. As an example, 5.9\% of CD fibrils had spacing of $66 \mathrm{~nm}$ or smaller, versus $2.6 \%$ for ZDSD. This disparity grew to $10.5 \%$ versus $6.1 \%$ by $66.5 \mathrm{~nm}$ and was maximized near $67.5 \mathrm{~nm}$ ( $40 \%$ for $\mathrm{CD}$ versus $27.8 \%$ for $Z D S D)$. This observation indicates fewer ZDSD fibrils with small spacing values versus CD. Between $5 \%$ and $45 \%$, the ZDSD population was shifted upward by an average of 0.3-0.4 nm. Eventually, the ZDSD population catches up (near $68.3 \mathrm{~nm}$ or at $73 \%$ of the population). Beyond $68.5 \mathrm{~nm}$, there were $19.1 \%$ of CD fibrils versus $15 \%$ for ZDSD indicating that in this range, there were also fewer ZDSD fibrils with larger spacing.

\section{Mechanical Changes in ZDSD Tail Tendon Fibrils}

Nanoscale mechanical changes were assessed using AFM. On average, 70 individual fibrils were indented from each animal (a subset of fibrils used for D-spacing analysis) for approximately 300 indentations per animal. Elastic modulus measurements from a single animal were averaged to yield the mean value from that tail. Indentations from one ZDSD tail were excluded because of problems with the indentation curves preventing proper curve fitting. This issue reduced the number of samples in ZDSD from four to three. There was no significant difference $(\mathrm{p}=0.071)$ between groups $(\mathrm{CD}: 3.81 \pm 1.49 \mathrm{MPa}$, ZDSD: $7.06 \pm 2.63$ $\mathrm{MPa})$.

Comparisons were made by investigating the population of all indentations within each group, leading to a total samples size of $n=1530$ for CD and $n=913$ for ZDSD. Histograms and CDFs were produced for the modulus, and the distributions were significantly different $(p<0.001$, Figure 3$)$. The dramatically different shape and breadth of the distributions indicates that modulus in ZDSD fibrils was more variable and shifted to higher values, in comparison to the relatively uniform and narrow $\mathrm{CD}$ population which was centered closely to its population mean. For example, $75 \%$ of the modulus measures in the $\mathrm{CD}$ population were below 4.7 MPa versus only $37 \%$ for ZDSD.

A final set of mechanical investigations were performed by pooling indents made in a fibril to yield a single value for that fibril. For those fibrils where D-spacing was also measured, linear correlation analysis was performed between D-spacing and modulus $(n=267$ for CD and $\mathrm{n}=154$ for ZDSD). The relationship between $\mathrm{D}$-spacing and modulus was significant but weak in the ZDSD samples $\left(\mathrm{r}^{2}=0.04, \mathrm{p}=0.009\right)$. No relationship existed in the $\mathrm{CD}$ samples 
$\left(\mathrm{r}^{2}=0.0013, \mathrm{p}=0.556\right)$. When all fibrils from both groups were considered, there was a significant but weak relationship $\left(\mathrm{r}^{2}=0.013, \mathrm{p}=0.021\right)$.

\section{Microscale Changes in ZDSD Tail Tendon Fascicles}

Fascicles from CD $(n=5)$ and ZDSD tails $(n=4)$ were mechanically tested to failure in tension. The fascicles had an average gauge length of $65 \pm 9 \mathrm{~mm}$, with no difference between groups. The average fascicle diameter was $447 \pm 51 \mu \mathrm{m}$ for CD and $386 \pm 36 \mu \mathrm{m}$ for ZDSD $(\mathrm{p}=0.190)$. At the fascicle level, no mechanical properties differed between groups (i.e. force, deformation, work; Table 2). At the material level, ultimate stress ( $\mathrm{p}=0.016)$ and modulus ( $\mathrm{p}=0.016$ ) were significantly greater in the ZDSD samples. No other differences reached significance.

\section{Relationship between Nanoscale and Microscale Parameters}

To relate properties measured at the nanoscale and microscale, Pearson product-moment correlations were calculated (Table 3). All measurements within each tail were averaged to yield the mean value from each property, resulting in five $C D$ values and four ZDSD values $(\mathrm{n}=3$ for ZDSD nanoscale modulus as noted above). No significant relationships existed between $\mathrm{D}$ spacing and any other property. The same was not true for nanoscale modulus, which had a strong and significant relationship with fiber yield stress $\left(p=0.050, r^{2}=0.497\right)$, and ultimate stress $\left(\mathrm{p}=0.022, \mathrm{r}^{2}=0.612\right.$ ). Most importantly, nanoscale modulus was strongly and significantly correlated with microscale modulus $\left(\mathrm{p}=0.04, \mathrm{r}^{2}=0.531\right)$ suggesting that nanoscale modulus could explain up to $53 \%$ of the variance in microscale modulus (Figure $4)$.

\section{DISCUSSION}

This study investigated multiscale mechanical impacts of a T2D-like condition in the rat tail tendon. Although changes noted are specific to tail tendon, similar results were noted in the bone of ZDSD rats indicating that changes are not restricted to tendon (unpublished data). With dietary alteration, ZDSD rats develop hyperglycemia, similar to the T2D condition in humans (Reinwald et al. 2009). A previous study in ZDSD bone demonstrated decreased mineral density and structural mechanical properties in the femur and L4 vertebral bodies, in addition to decreased tissue strength, modulus and toughness (Reinwald et al. 2009). A more recent study utilizing a novel testing technique known as Reference Point Indentation (RPI) showed that cortical bone from male ZDSD rats had higher indentation depth increase (IDI), a property that is inversely proportional to toughness (Gallant et al. 2012). The current study showed that tendon fascicles from female ZDSD rats had no differences in whole fascicle mechanical properties but had increased material-level strength and stiffness. At the nanoscale, the fibril D-spacing distribution was narrower and shifted towards higher spacing values in ZDSD. The distribution of nanoscale modulus was also shifted higher in ZDSD, but neither mean D-spacing nor mean modulus were significantly different than CD controls. Correlations between nanoscale and microscale properties indicate a direct relationship between the length scales, most notably in the positive and significant relationship between nanoscale and microscale modulus (Figure 4). It is possible that the changes seen here are due to the presence of AGEs, as noted in other rodent models of T2D (Tomasek et al. 1994; Odetti et al. 2000). However, AGEs were not directly quantified in the current study.

This study is a unique multiscale investigation of tendon structure and function. A previous study used AFM to image nanoscale features of collagen fibrils in diabetic rat tendon (Odetti et al. 2000). A limitation was that imaging took place in dried fibrils. As shown in prior studies of tendon collagen, morphology and mechanical properties are altered when collagen 
dries (Kemp et al. 2012; Wallace et al. 2012). All tests here took place in fluid. In other studies using this technique to quantify collagen nanoscale morphology, shifts in the Dspacing distribution have been noted following estrogen deficiency (Wallace et al. 2010b; Warden et al. 2013) and with Osteogenesis Imperfecta (Wallace et al. 2011; Kemp et al. 2012) in multiple tissues, but not between different tissue types in healthy mice (Wallace et al. 2010a), or between male and female mice. This study demonstrated that diabetic tendons had a distribution of fibril D-spacings that was narrower versus control (Figure 2). This observation is supported by data showing both ends of the ZDSD population were smaller versus $C D$, with far fewer small-spacing fibrils as well as fewer fibrils with large spacing values. Results also show that the bulk of the ZDSD population was shifted to higher values over the majority of its population. These data are consistent with another study which showed a shift in the D-spacing distribution along with increased AGEs in diabetic and glucose-incubated tendon samples (Odetti et al. 2000). AGEs in tendon were not quantified here, but the early glycation product HbA1c, a measure of glycated hemoglobin and an indicator of AGEs in the blood, was elevated by more than 2.5 fold in ZDSD rats (Table 1). Another study failed to show any change in D-spacing with ribose treatment in vitro (Tanaka et al. 1988). That study, as with many others, performed bulk measurements of collagen. As noted by the lack of mean difference in D-spacing here, bulk measurement could miss important structural information obtained by investigating individual fibrils.

Tensile mechanical properties were measured in tendon fascicles. Although other studies have indicated that differential swelling of fascicles can occur when stored in PBS prior to testing and can influence mechanical properties (Koob 1989; Screen et al. 2006; Fessel and Snedeker 2009), a swelling study indicated that this factor did not significantly contribute to the mechanical differences noted here (data not shown). The average diameter of ZDSD fascicles was not significantly different than $\mathrm{CD}$, but tended to be smaller. No structural mechanical properties differed between the groups (Table 2). This finding indicates that material-level properties compensated for reduced fiber size in ZDSD tails, maintaining whole-fiber properties at control levels. At the material-level, stiffness and strength were greater in ZDSD fibers, but at the expense of marginally decreased total strain to failure.

A goal of this study was to put functional meaning behind fibril D-spacing and to relate nanoscale and microscale properties. D-spacing of individual fibrils was regressed against the modulus obtained by pooling the 4-5 indents made on that fibril. The relationship in ZDSD was weak, and no relationship existed in $\mathrm{CD}$, suggesting no meaningful relationship between these properties. A similar finding was shown in tendons from normal mice and mice from the Brtl model of Osteogenesis Imperfecta (Kemp et al. 2012). One reason for a weak relationship between D-spacing and modulus may be because of the tight range of values found for D-spacing ( 64.1 to $71.2 \mathrm{~nm}$, a total range of $7.1 \mathrm{~nm}$ ). For all 1004 fibrils measured, the mean \pm standard deviation was $67.8 \pm 0.9 \mathrm{~nm}$. This data range may also be the reason that mean D-spacing from each animal failed to have a significant relationship with microscale mechanical measures (Table 3). It is also possible that D-spacing has no direct relationship with mechanical function in tendon fibrils or fibers, but further investigations need to be performed.

A weakness of this study's design is that properties were measured in different orientations at the different length scales due to technical limitations. While it is true that some groups have tested individual fibrils in tension using AFM (van der Rijt et al. 2006; Yang et al. 2012), the technique is challenging, requires special processing and would be impractical to get samples sizes large enough for relevant statistical comparisons. Another concern is the way values were pooled for regressions. At the nanoscale, each data point represented hundreds of individual indents performed in a tail and pooled. At the microscale, each value was the composite of the 10-12 fascicles tested in that animal. Each nanoscale and 
microscale value has a known and measured variance. As an example, one of the data points had a microscale modulus of $738 \pm 102 \mathrm{MPa}$ and an indentation modulus of $6.3 \pm 3.3 \mathrm{MPa}$. With the level of variance associated with each data point, it may be challenging to obtain strong correlations. In addition, the overall sample sizes were small for these comparisons ( $n=5$ in CD, $n=4$ in ZDSD with the exception of $n=3$ for nanoscale modulus). Despite these weaknesses, nanoscale indentation modulus was positively and significantly correlated with several microscale measures. The link between indent modulus and microscale strength/ stiffness is exciting and suggests that the stiffening of fascicles noted in the ZDSD rats has its roots at the nanoscale.

In conclusion, data from this study demonstrated nanoscale changes in collagen morphology and stiffening of individual fibrils in ZDSD tail tendons. Material-level strength and stiffness were also increased in ZDSD. Despite differences in nanoscale and microscale mechanical properties, ZDSD rats had no differences in whole fascicle mechanical properties. Strong and significant relationships existed between nanoscale and microscale mechanical measures indicating that diabetes-induced changes in material strength and modulus were driven by alterations at the nanoscale.

\section{Acknowledgments}

Funding Sources: This work was supported by IUPUI departmental start-up funds (JMW), Research Support Funds Grant from the IUPUI Office of the Vice Chancellor for Research (JMW) and NIH grant AR-047838 (DBB).

\section{REFERENCES}

Canty EG, Kadler KE. Procollagen trafficking, processing and fibrillogenesis. Journal of Cell Science. 2005; 118(7):1341-1353. [PubMed: 15788652]

Centers for Disease Control and Prevention. US Department of Health and Human Services, Centers for Disease Control and Prevention; Atlanta, GA: National diabetes fact sheet: national estimates and general information on diabetes and prediabetes in the United States, 2011.

Couppe C, Hansen P, Kongsgaard M, Kovanen V, Suetta C, Aagaard P, Kjaer M, Magnusson SP. Mechanical properties and collagen cross-linking of the patellar tendon in old and young men. Journal of applied physiology. 2009; 107(3):880-886. [PubMed: 19556458]

Duquette J, Grigg P, Hoffman A. The effect of diabetes on the viscoelastic properties of rat knee ligaments. Journal of Biomechanical Engineering. 1996; 118(4):557-564. [PubMed: 8950660]

Erickson B, Fang M, Wallace JM, Orr BG, Les CM, Banaszak Holl MM. Nanoscale structure of type I collagen fibrils: Quantitative measurement of D-spacing. Biotechnology Journal. 2013; 8:117-126. [PubMed: 23027700]

Fang M, Liroff KG, Turner AS, Les CM, Orr BG, Holl MMB. Estrogen Depletion Results in Nanoscale Morphology Changes in Dermal Collagen. Journal of Investigative Dermatology. 2012; 132:1731-1797.

Fessel G, Snedeker JG. Evidence against proteoglycan mediated collagen fibril load transmission and dynamic viscoelasticity in tendon. Matrix Biology. 2009; 28(8):503-510. [PubMed: 19698786]

Fox AJ, Bedi A, Deng XH, Ying L, Harris PE, Warren RF, Rodeo SA. Diabetes mellitus alters the mechanical properties of the native tendon in an experimental rat model. Journal of Orthopaedic Research. 2011; 29(6):880-885. [PubMed: 21246619]

Fratzl, P. Collagen: Structure and Mechanics. Springer; 2008.

Gallant MA, Brown DM, Organ JM, Allen MR, Burr DB. Reference-point indentation correlates with bone toughness assessed using whole-bone traditional mechanical testing. Bone. 2012; 53(1):301305. [PubMed: 23274349]

Hodge, AJ.; Petruska, JA. Aspects of Protein Structure. Academic Press; New York: 1963. Recent Studies with the Electron Microscope on Ordered Aggregates of the Tropocollagen Molecule; $p$. 289-306.G. N. Ramachandran 
Kastelic J, Galeski A, Baer E. The multicomposite structure of tendon. Connective tissue research. 1978; 6(1):11-23. [PubMed: 149646]

Kemp A, Harding C, Cabral WA, Marini JC, Wallace JM. Effects of Tissue Hydration on Nanoscale Structural Morphology and Mechanics of Individual Type I Collagen Fibrils in the Brtl Mouse Model of Osteogenesis Imperfecta. Journal of Structural Biology. 2012; 180(3):428-438. [PubMed: 23041293]

Knott L, Bailey AJ. Collagen cross-links in mineralizing tissues: a review of their chemistry, function, and clinical relevance. Bone. 1998; 22(3):181-187. [PubMed: 9514209]

Koob TJ. Effects of chondroitinase-ABC on proteoglycans and swelling properties of fibrocartilage in bovine flexor tendon. Journal of Orthopaedic Research. 1989; 7(2):219-227. [PubMed: 2493083]

Launey ME, Buehler MJ, Ritchie RO. On the mechanistic origins of toughness in bone. Annual Review of Materials Research. 2010; 40:25-53.

Li Y, Fessel G, Georgiadis M, Snedeker JG. Advanced glycation end-products diminish tendon collagen fiber sliding. Matrix Biology. 2013; 32(3-4):169-177. [PubMed: 23348249]

Merlotti D, Gennari L, Dotta F, Lauro D, Nuti R. Mechanisms of impaired bone strength in type 1 and 2 diabetes. Nutrition, Metabolism and Cardiovascular Diseases. 2010; 20(9):683-690.

Odetti P, Aragno I, Rolandi R, Garibaldi S, Valentini S, Cosso L, Traverso N, Cottalasso D, Pronzato M, Marinari U. Scanning force microscopy reveals structural alterations in diabetic rat collagen fibrils: role of protein glycation. Diabetes/metabolism research and reviews. 2000; 16(2):74-81. [PubMed: 10751746]

Orgel JPRO, Irving TC, Miller A, Wess TJ. Microfibrillar structure of type I collagen in situ. Proceedings of the National Academy of Sciences. 2006; 103(24):9001-9005.

Reddy GK. Cross-linking in collagen by nonenzymatic glycation increases the matrix stiffness in rabbit achilles tendon. Experimental Diabetes Research. 2004; 5(2):143-153.

Reinwald S, Peterson RG, Allen MR, Burr DB. Skeletal changes associated with the onset of type 2 diabetes in the ZDF and ZDSD rodent models. American Journal of Physiology-Endocrinology And Metabolism. 2009; 296(4):E765-E774. [PubMed: 19158319]

Rigozzi S, Müller R, Stemmer A, Snedeker J. Tendon glycosaminoglycan proteoglycan sidechains promote collagen fibril sliding-AFM observations at the nanoscale. Journal of Biomechanics. 2012; 46(4):813-818. [PubMed: 23219277]

Screen HR, Chhaya VH, Greenwald SE, Bader DL, Lee DA, Shelton JC. The influence of swelling and matrix degradation on the microstructural integrity of tendon. Acta biomaterialia. 2006; 2(5): 505-513. [PubMed: 16839828]

Singh R, Barden A, Mori T, Beilin L. Advanced glycation end-products: a review. Diabetologia. 2001; 44(2):129-146. [PubMed: 11270668]

Svensson RB, Mulder H, Kovanen V, Magnusson SP. Fracture Mechanics of Collagen Fibrils: Influence of Natural Cross-Links. Biophysical journal. 2013; 104(11):2476-2484. [PubMed: 23746520]

Tanaka S, Avigad G, Brodsky B, Eikenberry EF. Glycation induces expansion of the molecular packing of collagen. Journal of Molecular Biology. 1988; 203(2):495-505. [PubMed: 3143838]

Tang S, Zeenath U, Vashishth D. Effects of non-enzymatic glycation on cancellous bone fragility. Bone. 2007; 40(4):1144-1151. [PubMed: 17257914]

Tomasek JJ, Meyers SW, Basinger JB, Green DT, Shew RL. Diabetic and age-related enhancement of collagen-linked fluorescence in cortical bones of rats. Life Sciences. 1994; 55(11):855-861. [PubMed: 8072386]

van der Rijt JA, van der Werf KO, Bennink ML, Dijkstra PJ, Feijen J. Micromechanical testing of individual collagen fibrils. Macromolecular bioscience. 2006; 6(9):697-702. [PubMed: 16967482]

Vashishth D, Gibson GJ, Khoury JI, Schaffler MB, Kimura J, Fyhrie DP. Influence of nonenzymatic glycation on biomechanical properties of cortical bone. Bone. 2001; 28(2):195-201. [PubMed: 11182378]

Wallace JM, Chen QS, Fang M, Erickson B, Orr BG, Holl MMB. Type I Collagen Exists as a Distribution of Nanoscale Morphologies in Teeth, Bones, and Tendons. Langmuir. 2010a; 26(10): 7349-7354. [PubMed: 20121266] 
Wallace JM, Erickson B, Les CM, Orr BG, Holl MMB. Distribution of type I collagen morphologies in bone: Relation to estrogen depletion. Bone. 2010b; 46(5):1349-1354. [PubMed: 19932773]

Wallace JM, Harding C, Kemp A. Effects of Hydration on Nanoscale Structural Morphology and Mechanics of Individual Type I Collagen Fibrils. Proceedings of the 2012 Spring Meeting of the Materials Research Society. 2012; 1465

Wallace JM, Orr BG, Marini JC, Banaszak Holl MM. Nanoscale Morphology of Type I Collagen is Altered in the Brtl Mouse Model of Osteogenesis Imperfecta. Journal of Structural Biology. 2011; (173):146-152. [PubMed: 20696252]

Warden SJ, Galley MR, Hurd AL, Wallace JM, Gallant MA, Richard JS, George LA. Skeletal Exercise in Young Female Rats Provides Lifelong Benefits to Cortical Bone Properties Independent of an Artifical Menopause. Endocrinology. 2013; 154(9):3178-3187. [PubMed: 23782938]

Yang L, van der Werf K, Dijkstra P, Feijen J, Bennink M. Micromechanical analysis of native and cross-linked collagen type I fibrils supports the existence of microfibrils. Journal of the Mechanical Behavior of Biomedical Materials. 2012; 6:148-158. [PubMed: 22301184] 


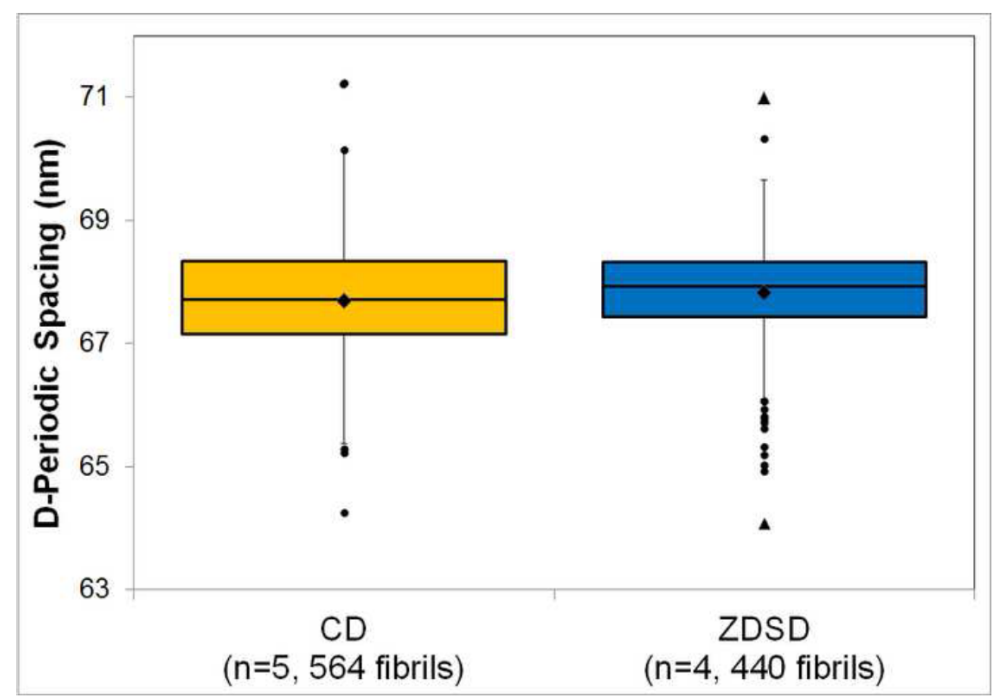

Figure 1. Boxplot representation of D Periodic Spacing Values

For each group, the box is the interquartile region (IQR) from all measured fibrils (middle $50 \%$ of the data), the horizontal line inside of the box is the median, and the diamond is the mean. The whiskers on the box represent the Q1 $-1.5^{*} \mathrm{IQR}$ and $\mathrm{Q} 3+1.5 * \mathrm{IQR}$ for that group. Values between 1.5*IQR and 3*IQR away from Q1 or Q3 are mild outliers (shown as circles) and those more than $3 * \mathrm{IQR}$ from Q1 or Q3 are major outliers (shown as triangles). Outliers were included in all analyses. When the mean values from the five $C D$ samples were compared with the four values from the ZDSD group, no difference was noted $(\mathrm{p}=0.645)$. 

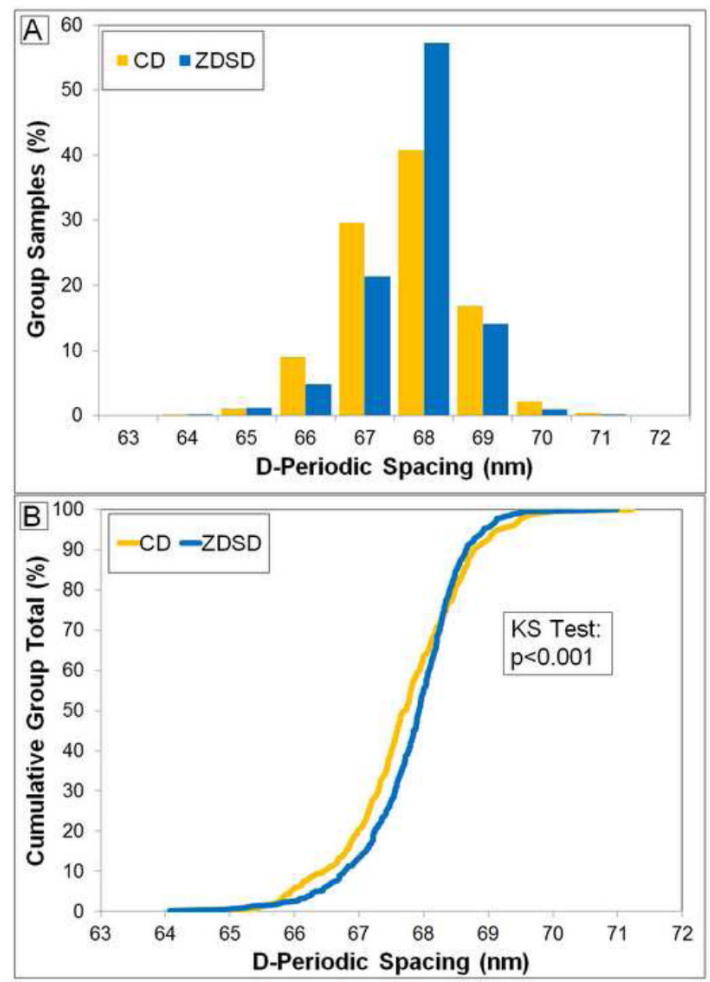

Figure 2. Histogram and Cumulative Distribution Function for D-Periodic Spacing Panel A shows the histogram and panel B shows the Cumulative Distribution Function (CDF) computed from all measurements in each group ( $n=564$ for $C D, n=440$ for ZDSD). Apparent in both panels, the ZDSD population was shifted upward to higher spacing values in comparison to their CD counterparts, particularly in the lower half of the data range. A Kolmogorov-Smirnov test indicated a significant difference in the populations. 

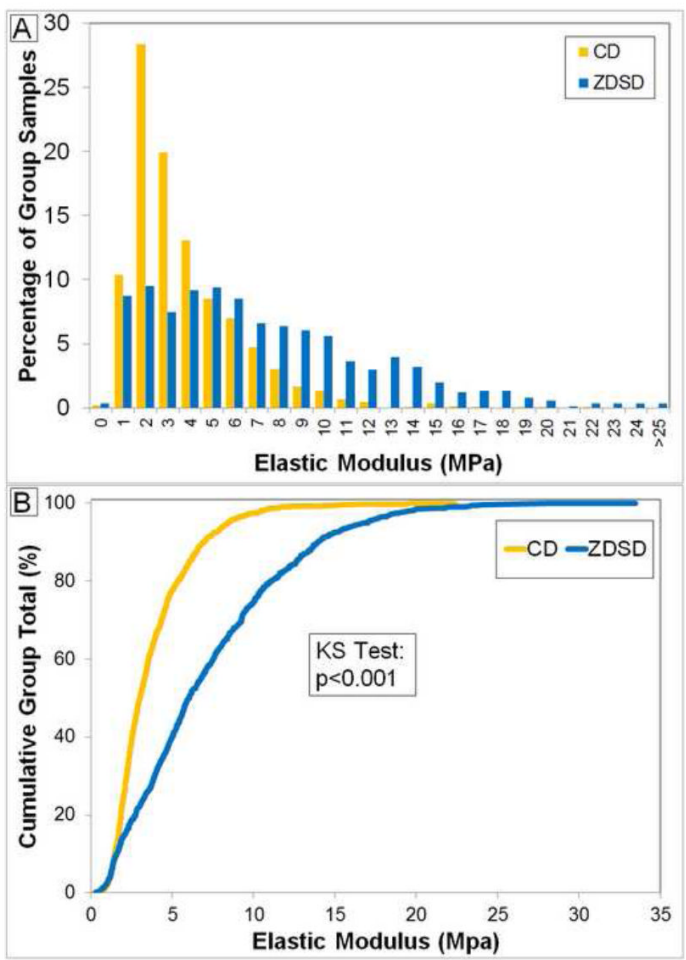

Figure 3. Histogram and Cumulative Distribution Function for Nanoscale Elastic Modulus Panel A shows the histogram and panel B shows the Cumulative Distribution Function (CDF) computed from all nanoscale modulus measurements in each group $(n=1530$ for CD, $\mathrm{n}=913$ for ZDSD). The modulus distribution was significantly different between the two groups $(\mathrm{p}<0.001)$. The graphs indicate that modulus in the ZDSD fibrils was shifted higher and was more variable compared with the $\mathrm{CD}$ population. 


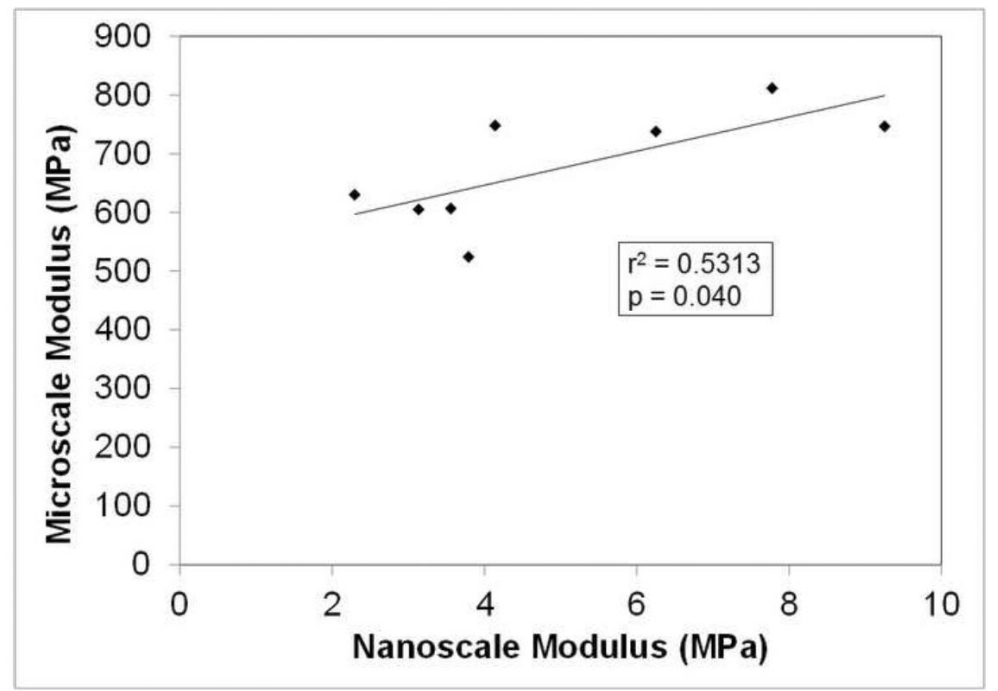

Figure 4. Linear Correlation Between Nanoscale Modulus and Microscale Modulus All measurements within each tendon sample were averaged to yield the mean value from each property for that tail, resulting in five CD values and four ZDSD values ( $n=3$ for ZDSD nanoscale modulus as noted in the text). Nanoscale modulus had a strong and significant relationship $(\mathrm{p}=0.040)$ with microscale modulus. 
Table 1

Animal Information $(n=5 \mathrm{CD}, \mathrm{n}=4$ ZDSD)

\begin{tabular}{|r|c|c|c|}
\hline & CD & ZDSD & p value \\
\hline Baseline Body Weight (g) & $302 \pm 34$ & $340 \pm 14$ & 0.190 \\
\hline Final Body Weight (g) & $433 \pm 89$ & $411 \pm 30$ & 0.730 \\
\hline Baseline Glucose (mg/dl) & $126 \pm 8$ & $114 \pm 16$ & 0.286 \\
\hline Final Glucose (mg/dl) & $\mathbf{1 3 1} \pm \mathbf{1 6}$ & $\mathbf{4 7 2} \pm \mathbf{3 8}$ & $\mathbf{0 . 0 1 6}$ \\
\hline Baseline HbA1c (\%) & $4.2 \pm 0.3$ & $4.4 \pm 0.1$ & 0.250 \\
\hline Final HbA1c (\%) & $\mathbf{3 . 9} \pm \mathbf{0 . 1}$ & $\begin{array}{c}\mathbf{1 0 3} \pm \\
\mathbf{0 . 9}\end{array}$ & $\mathbf{0 . 0 1 6}$ \\
\hline
\end{tabular}

Values are presented as mean \pm standard deviation 
Table 2

Microscale Mechanical Properties ( $n=5$ CD, $n=4$ ZDSD)

\begin{tabular}{|r|c|c|c|c|c|}
\hline & \multicolumn{2}{|c|}{ CD } & \multicolumn{2}{c|}{ ZDSD } & p value \\
\hline & Mean & Std. dev. & Mean & Std. dev. & \\
\hline Yield Force (N) & 2.20 & 0.18 & 1.92 & 0.47 & 0.556 \\
\hline Ultimate Force (N) & 4.54 & 0.33 & 4.56 & 0.99 & 0.730 \\
\hline Yield Deformation (microns) & 1542 & 129 & 1664 & 103 & 0.190 \\
\hline Post-Yield Deformation & 4391 & 460 & 4253 & 375 & 0.905 \\
\hline (microns) & & & & & \\
\hline Failure Deformation (microns) & 5929 & 509 & 5916 & 456 & 0.905 \\
\hline Pre-Yield Work (mJ) & 1.78 & 0.28 & 1.69 & 0.42 & 0.730 \\
\hline Post-Yield Work (mJ) & 17.24 & 2.84 & 15.65 & 4.66 & 0.905 \\
\hline Total Work (mJ) & 19.02 & 3.04 & 17.33 & 5.07 & 0.905 \\
\hline Yield Stress (MPa) & 14.18 & 2.56 & 16.19 & 1.01 & 0.286 \\
\hline Ultimate Stress (MPa) & $\mathbf{2 9 . 2 6}$ & $\mathbf{4 . 9 7}$ & $\mathbf{3 8 . 4 7}$ & $\mathbf{1 . 6 2}$ & $\mathbf{0 . 0 1 6}$ \\
\hline Yield Strain (\%) & 2.57 & 0.36 & 2.43 & 0.18 & 0.286 \\
\hline Failure Strain (\%) & 9.83 & 0.93 & 8.65 & 0.87 & 0.190 \\
\hline Modulus (MPa) & $\mathbf{6 2 0 . 8}$ & $\mathbf{7 6 . 8}$ & $\mathbf{7 6 7 . 2}$ & $\mathbf{2 9 . 8}$ & $\mathbf{0 . 0 1 6}$ \\
\hline Pre-Yield Toughness (MPa) & 0.19 & 0.05 & 0.21 & 0.02 & 0.413 \\
\hline Total Toughness (MPa) & 2.00 & 0.50 & 2.07 & 0.31 & 0.413 \\
\hline & & & & \\
\hline
\end{tabular}


Table 3

Correlations Between Nanoscale and Microscale Properties

\begin{tabular}{|c|c|c|}
\hline & $\mathbf{r}^{2}$ value & p value \\
\hline Indent Modulus vs. Yield Stress & $\mathbf{0 . 4 9 7}$ & $\mathbf{0 . 0 5 0}$ \\
\hline Indent Modulus vs. Ultimate Stress & $\mathbf{0 . 6 1 2}$ & $\mathbf{0 . 0 2 2}$ \\
\hline Indent Modulus vs. Yield Strain & 0.013 & 0.784 \\
\hline Indent Modulus vs. Failure Strain & 0.021 & 0.732 \\
\hline Indent Modulus vs. Fiber Modulus & $\mathbf{0 . 5 3 1}$ & $\mathbf{0 . 0 4 0}$ \\
\hline Indent Modulus vs. Toughness & 0.429 & 0.078 \\
\hline Indent Modulus vs. D Spacing & $<0.001$ & 0.999 \\
\hline D Spacing vs. Yield Stress & 0.200 & 0.228 \\
\hline D Spacing vs. Ultimate Stress & 0.091 & 0.429 \\
\hline D Spacing vs. Yield Strain & 0.398 & 0.068 \\
\hline D Spacing vs. Failure Strain & 0.025 & 0.686 \\
\hline D Spacing vs. Fiber Modulus & 0.005 & 0.859 \\
\hline D Spacing vs. Toughness & 0.023 & 0.699 \\
\hline
\end{tabular}

